\title{
Beiträge zur Kenntniss der Refractions- und Accommodations-Anomalien.
}

\author{
Yon \\ F. C. Donders.
}

Einfluss des Lebensalters auf $A$ ccommodation und Refraction.

Mit dem zunehmeriden Lebensalter erleidet das Auge eine Reihe Veränderungen verschiedener Art. Siu sind zum Theile schon bei auswendiger Beobachtung zu erkennen, wie der geringere Glanz der Cornea und Conjunctiva, die kleinere Pupille, die geringere Durchsichtigkeit von Sclerotica und Iris, die geringere Tiefe der Augenkammer, der arcus senilis u. s. w. Zum Theile aber werden sie erst bei der eigentlichen anatomischen Zergliederung sichtbar. Dahin gehören z. B.: die Wucherung der hyaloiden Membranen mit secundären Veränderungen der Retina, die Kalkconcremente in dem hinteren Theile der sclerotica, die eigenthümliche Metamorphose des vorderen Theiles der sclerotica, Veränderungen der choroidea, Atrophie des m. Brückianus, grössere Festigkeit und mehr gelbliche Farbenuance der Linse mit darauffolger Trübung einiger Lagen und geringerer Klarheit des Glaskörpers. Noch ehe die 
anatomische Untersuchung eine Spur von Trübung darthun kann, lehrt die vergleichende ophthalmoskopische Untersuchung von gesunden Augen in verschiedenem Lebensalter, dass die vollkommene Klarheit und Durchsichtigkeit mit dem Steigen der Jahre verloren geht, während der fundus oculi des Kindes so wunderschön hell und klar wahrzunehmen ist.

Die anatomischen Veränderungen bedingen verschiedene Functionsstörungen. Wir wollen hier nur diejenigen behandeln, welche sich auf die Accommodation und Refraction des Auges beziehen. Alle anderen, sowic die sie bedingenden anatomischen Veräuderungen übergehen wir hier.

Zuerst wollen wir über die Modification des Accommodations.Vermügens handeln, welche schon lange aufgetreten ist, ehe der Refractionszustand des Auges in der Ruhe einige Veränderung erfahren hat; die Entfernung des fernsten Punktes nämlich hleibt lange Zeit unverändert, während die des nächsten Punktes des deutlichen Sehens allmälig grösser und grösser wird. So nimmt mithin die Accommodationsbreite ab. Die zunehmende Entfernung des nächsten Punktes ist ein allgemein bekanntes Factum. Man verfällt aber in einen Irrthum, wenn man annimmt, dass dieses Entfernen des nächsten Punktes erst seit dem 40. Jahre anfängt. In diesem Alter wird dieses Fernerrücken des nächsten Punktes, unter gewissen Umständen, erst als Störung für das normale Auge bemerkt, und darum wird man dann erst auf die sogenannte Schwäche des Auges autmerksam; in Wahrheit entfernt sich aber der nächste Punkt des deutlichen Sehens schon in den Jünglingsjahren, ja schon vor der Pubertät.

Diese Veränderung trifft alle Angen ohne Unterschied, sowohl das myopische (wenn es anders gesund bleiht) als auch das hypermetropische und das emme- 
tropische Auge. Den Verlauf des năchsten Punktes in jedem dieser Augen werden wir später jeden für sich verfolgen. Hier möge nur norh die Frage aufgeworfen werden, wie und wodurch das Accommodationsvermögen, das auf Muskelwirung beruht, in so jugendlichem Alter schon an Breite verliert, während alle Functionen und namentlich die Muskelkraft in fortschreitender Entwickelung sich befinden. Der Musc. Briickianus ist normal geblieben und mit voller Kraft wirksam. Da dieses unwandelbar fest steht, so kommt man wie von selbst zu der Annahme, doch wenigstens im Arifange die Abnahme nur in den Theilen zu suchen, welche bei der Accommodation passiv verändert-werden, nicht aber in denjenigen, welche durch ihre Acti-. vität die Accommodation einleiten. Das passiv veränderte Organ nun ist die Linse. Ist nun wirklich in ihr ein Erklärungsgrund für das schon so frühzeitige Abnehmen der Accommodationsbreite zu finden? Man weiss, dass die Festigkeit der Linse in höherem Alter viel grösser ist, als in der Jugend. Es ist nir aber überdies vorgekommen, dass die Festigkeit schon in jugendlichem Alter im Zunehmen begriffen ist. Es ist aber leicht zu begreifen, dass dieselbe Muskelwirkung dann nicht mehr dieselbe Veränderung in der Form der Linse hervorrufen kann. Das frühzeitige Abnehmen der Accommodationsbreite ist daher höchst wahrscheinlich so zu erklären.

Eine geringe Abnahme der Refraction kommt erst allmälig zu. Stande, nachdem das Accommodationsvermögen schon bedeutend abgenommen hat. Es geht daraus hervor, dass jetzt auch der Fernpunkt des deutlichen Sehens anfängt, sich mehr von dem Auge zu entfernen, dass mithin der hintere Brennpunkt tiefer im Auge oder sogar hinter der Netzhaut gelegen ist. Wie schon erwähnt, iat aber diese Abnahme der Re- 
lrartion erst in höherem Alter wahrzunehmen. Mil rem 40. Jahre hat sie noch nich oder kaum angefangen und rest im 55. oder 60. Jahre ist sie in einem ursprïnglich emmetropischen Auge deutlich vorhanden; bei der geringeren Accommodationsbreite kann alsdann bei parallelen Sehlinien sogar nicht mebr für parallele Strahlen açcommodirt werden, und ein positives Glas wird auch filr die Ferne nöthig. Es besteht aber hierin ein 'grosser Unterschied bei den verschiedenen Individuen: die Abuahme der Refraction ist of so gering, dass es Mühe kostet, sie zu constatiren; mitunter scheint sie ganz zu fehlen; in anderen Fällen dagegen ist sie ziemlich bedentend.

Welche Ursache bedingt diese Abnabme der Refraction, welche die senile Veränderung des Auges begleitit?

Wenn man die grossen individuellen Unterschiede berücksichtigt, so würde man darin Veranlassung finden könuen, zu fragen, ob nicht diese Abnahme in: Wirklichkeit nur scheinbar ist, ob nämlich in denjenigen Fällen, in denen in höherem Alter Hypermetropie deutlich wahrnehmbar wird, nicht schon in der Jugend eine ebenso bedeutende latente Hypermetropie vorhanden war. Wenn sich die Sache wirklich so verhielte, so würde, wie man leicht einsieht, die Veränderungr durchaus auf Abnahme des Accommodationsvermögens zurü.:kgebracht werden mï̈ssen. Diese Frage darf aber mit vollem Rechte verneint werden. Mitunter entwickelt sich die verminderte Refraction in relativ kurzer Zeir, namentlich dann, wenn Spuren von Trübung entstehen; auch bei Myopie hahe ich Gelegenheit gehabt, einigo Male Abnahme der Refraction zu constatiren. Ich wiederhole daber die Frage nach der Ursache dieser Erscheinung. Man hat Abflachung der Cornea und verminderten Umfang des Augapfels, dessen Gesichtsaxe 
dabei kürzer geworden, zu Hülfe gerufen.: Es kommint mir aber wahrscheinlich vor, dass die Ursache in del Linse zu finden "sein wird. Siè rückt im hölieren Alter zusammen mit der Iris etwas nach vorne, und dies veranlasst das scheinbare Flacherwerden der Ilornhaut. Diese Ortsveränderung der Linse wïrde aber an und für sich gerade die entgegengesetzto Wirkung zur Folge haben, das heisst, sie würde den Brennpünkt cinigermassen nach vorne hin versetzen. Auch dieser Einfluss muss, wenn die Refraction anders atinekmen söll, deshálb durch eine besondere Modification neutralisirt werden. Sie ist, wenn ich nicht irre, namentlich in eirer mehr gleichmässigen Festigkeit der verschiedenen Schichten der Linse zu suchen. Schon Thomas Young bemerkte, was Senff, Listing und Andere mehr ausgeführt haben, dass die Linse mit ihrem lamellären Baue wegen des geringeren lichtbrechenden Vermögens : ihrèr äusseren Schichten eine kürzere Brennweite hat, als cine Lińse von derselben Form haben wïrde, die ganz aus einer Substanz zusammengesetzt wäre, welche das dem Kerne der Linse eigenthümliche Lichtbrechungsvermügen besässe. Wenn mithin die äusseren Schichten mit zunebmendem Alter fester werden, so muss dalurch die Brennweite grüsser werden. Für dieses Festerwerden spricht nun namentlich die in höherem Alter zunehmende Reflexion der vorderen und hinteren Fläche der Linse, eine Reflexion, welche dem Unterschiede im lichtbrechenden Vermögen zwischen den äusseren Schichtevi der Linse und dem humor aqueus und dem Glaskörper proportionir ist. Ueberdies scheint die Linse im höheren Alter flacher zu werden, weshalb die Krümmungsstrahlen ihrer Oberfläche grösser werden. Von dem Flacherwerden der Cornea und dem Kürzerwerden der Gesichtsaxe habe ich mich aber andererseits nicht überzeugen können und darum glaube ich, dass die Abnahme 
der Refraction ausschliesslich durch Veränderungen der Linse bedingt sein muss. Dafür spricht auch der Umstand, dass dic Abnahme der Refraction schliesslich gleichen Schritt hält mit der Abnahme des Accommodationsvermögens; dies deutet doch auf einen für beide gemeinschaftichen Ursprung und oben haben wir geseben, dass letztere hauptsächlich in einem Härterwerden der Linse ihren Grund hat. Den Glaskörper habe ich nicht in Bezug auf sein lichtbrechendes Vermögen in verschiedenem Alter untersucht. Es versteht sich von selbst, dass, da seine Vorderfläche concav ist, eine Zunahme seines lichtbrechenden Vermögens den hinteren Brennpunkt des Auges $n$ ach hinten verschieben würde.

Die Veränderungen der Accommodation und Refraction kommen, wie ich schon oben bemerkt, bei jeder Form des Auges vor. Die Behandlung derselben in dem emmetropischen, in dem myopischen, sowie in dem hypermetropischen Auge, jede für sich wird uns Gelegenheit geben, verschiedene Einzelheiten näher zu betrachten.

Fig. IV stellt den Verlauf des Nahe; und Fernpunktes, und mithin den des Accommodationsvermögens im emmetropischen Auge bei verschiedenem Alter vor.

Die auf der linken Seite befindlichen Ziffern geben, wie früher, die Entfernung (in Pariser Zoll) an, für welche accommodirt werden kann; diejenigen, welche unterhalb $\infty$ vorkommen, haben einen negativen Werth, gerade wie in den früheren Figuren; sie geben die Entfernung an, in welcher die convergirenden Strahlen, für welche das. Auge accommodirt ist, hinter dem Knotenpunkte zur Vereinigung kommen; $\boldsymbol{p} \boldsymbol{p}^{\prime}$ giebt den Verlauf des Nahepunktes, $r r^{\prime}$ den des Fernpunktes 


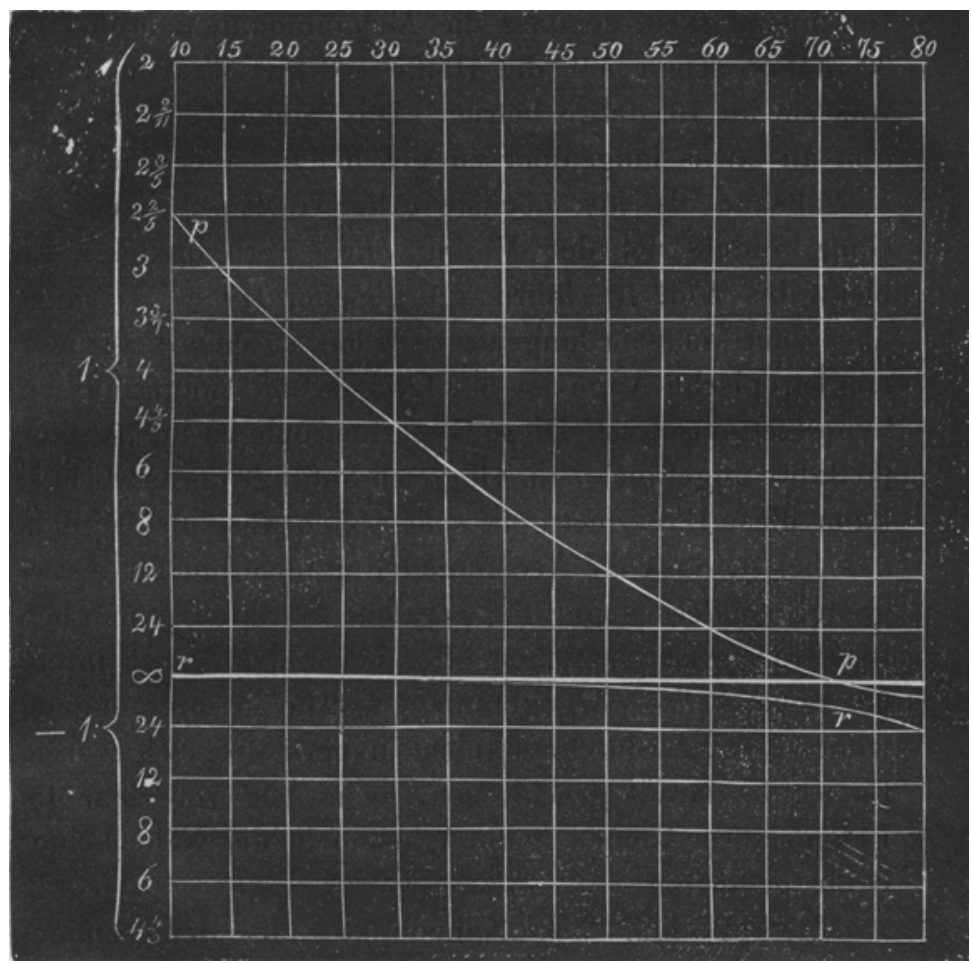

Figur IV.

an; die oberhalb der Figur vorkommenden Ziffern weisen die verschiedenen Lebensjahre an. Auf den Linien $p p^{\prime}$ und $r r^{\prime}$ kann man daher für jedes Luebensjahr den Nahe und Fernpunkt des deutlichen Sebens ablesen, indem die jedesmalige Entternung dieser zwei Linien von einander die Accommodationsbreite kennen lehrt: die Entfernung von zwei horizontalen Linien . ist nämlich wiederum $=1 /$ Accommodationsbreite. Aus der Figur geht unmittelbar hervor, dass schon von dem zehnten Jahre an, in welchem die Beobachtung möglich wird, der Nahepunkt dem Auge näher rückt und zwar mit ziemlich gleichmässiger 
Geschwindigkeit, so. dass die Accommodationshrcite im 30. Jahre schon auf die Hälfte reducirt ist von dem, was sie im 10. Jahre betrug. Das Sinken scheint von nun an etwas langsamer zu geschehen, aber doch unanfhaltsam bis ins böchste Alter fortzuschreiten. Ganz anders ist der Verlauf des Fernpunktrs. 'El' bleibt bis zum 40. Jahre auf' gleicher Höhe, - fängt aber dann an, sehr langsam zu fallen, so dass das früber emmetropische Auge im 50. Jahre schon einigermassen hypermetropisch ist. Diese Hypermetropie ist im 70. orler 80. Jahre ungetähr $==1 / 24$. Obgleich anfangs nur erworben, kann diese Hypermetropie später eine absolute werden, das heisst, dass die Accommodation nicht nur für divergente. sondern sogar fïr parallele Strablen unmöglich wird. Dies ist mir schon ofter bei Individuen in ihrem 60. Lebensjahre vorgekommen, welche in ihrer Jugend wahrscheinlich durchaus nicht hypermetropisch waren. Es wurde darauf geschlossen, wenn sie vor dem $4 \overline{5}$. Lebensjahre Abends beim Arbeiten in der Năhe keiner Brille bedurften.

Der Verlauf des Nahepunktes in dem emmetropischen Auge wurde aus einer grossen Zahl von Beobachtungen hergeleitet. In Fig. V. ist jede Beobachtung durch einen Punkt angegeben und die Lage dieser Punkte giebt zu gleicher Zeit an, dass die Abweichungen von dem durschnittichen Verlaufe nicht besonders gross sind. Zum Theile kommen diese zweifelsohne noch auf Rechnung von individuellen Fehlern; bei einigen Abweichungen kann auch ein geringer Grad von latenter Hypermetropie mit im Spiele sein. Beim Verfertigen dieser Figur wurden zum grössten Theile emmetropische Augen herücksichtigt; Myopie in geringem Grade (=-- 1:40 oder weniger) wurde mit benutzt. Ja es sind gerade diese myopischen Augen, welche den Vorzug verdienen, denn nur bei diesen hat man, ohne kunstmässige Lähmung der 


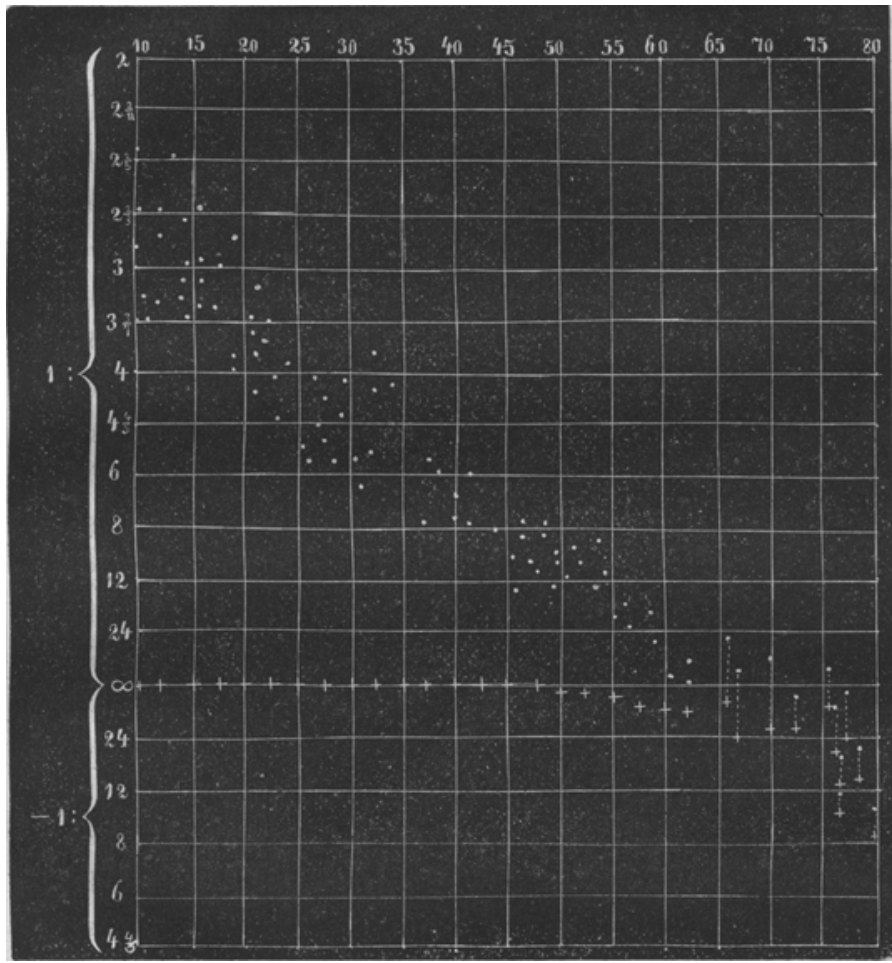

Figur V.

Accommodation, hinreichende Gewissheit, dass der Nahepunkt nicht durch eine latente Hypermetropie beeinträchtigt wird, und man darf wohl voraussetzen, dass bei diesen geringen Graden von Myopie die Accommodationsbreite von der des emmetropischen Auges nicht verschieden ist. Der Nahepunkt wurde natürlich im Verhältniss zur gefundenen Myopie reducirt. Endlich muss ich noch bemerken, dass der Nahepunkt, wenn er ferner als 8 " gefunden wurde, stets aus ciner mit positiven Gläsern gemachten Bestimmung berechnet wurde. Dadurchi wurde er auf die Entfernung vou ungefähr $8^{\prime \prime}$ gebracht. Wenn dies vernachlässigt wäre, so würde $A$, wegen 
der Abwesenheit der Convergenz in höherem Alter zu gering angeschlagen worden sein.

Viel zusammengesetzter und zugleich mehr auseinanderlaufend ist der Verlauf des Nahe- und Fernpunktes in dem myopischen $\Lambda$ uge. Die Myopie ist fast stets progressiv; sie ist dies aber in verschiedenem Grade. Während der'Verlauf des Fernpunktes hierinit direct und in verschiedenem Grade modificirt wird, erfährt der des Nahepunktes mittelbar eiue Veränderung. Den Verlauf der Myopie habe ich stets sorgfältig verfolgt. Dies hielt ich für sehr wichtig. Das bekannte Factum, dass Myopen bei schwachem Lichte kleine Gegenstände erkennen können, und vorzüglich der Umstand, dass sie in höherem Alter keine Brille brauchen, um in der Nähe zu sehen, haben dem Vorurtheile, dass myopische Augen als besonders starke Augen zu betrachten sind, fast allgemein Eingang verschafft. Sogar viele Aerzte haben sich dadurch irre führen lassen. Der Augenarzt hat aber leider nur zu oft Gelegenheit, sich von dem Gegentheile zu überzeugen. Ich sage es ohne Zaudern, dass ein myopisches Auge ein krankes $\Lambda$ uge ist. Dabei besteht mehr als eine einfache Anomalie der Refraction. Der optische Character möge hierin gelegen sein, der anatomische ist eine Ausdehnung des Auges mit Vcrlängerung, vorzüglich der Sehaxe, und meistens auch der anderen Axen, wiewohl in geringerem Grade. Wenn diese Ausdehnung eine gewisse Höhe erreicht hat, so sind die Membranen an dem hinteren Pole so sehr verdünnt, und der Widerstand hat so sehr abgenommen, dass die Ausdehnung hier nicht stationär bleiben kann, um so weniger, weil der Druck der Flüssigkeiten im myopischen Auge meistens etwas zugenommen hat. In dieser fortschreitenden Ausdehnung ist die Progressivität der 
Myopie, welche eine wahre Kránkheit des Auges ist, gelegen.

Nach den Obigen ist es leicht zu begreifen, dass hohe Grade von Myopic nicht so leicht stationär bleiben, als geringe; in höherem Alter fahren sie selbst fort, sich unter zunehmender Atrophie zu entwickeln. In der Jugend ist vielleicht jede Mycopie mehr oder weniger progressiv; das Fortschreiten des Uebels ist dann von Irritationserscheinungen begleitet, welche, wie vi $G$ i äfe lehrte, sogar den Character von sclerotico-choroiditis posterior annehmen können. Dies ist die kritische Periode für das myopische Auge: wenn die Myopie dabei nicht zu sehr zunimmt; danu kann sie stationär bleiben und in hölierem Alter sugar abnebmen; wenn sie sich aber in hohem Grade entwickelt, so ist das Fortschreiten später schwerlich zu hemmen. In dieser Periode hat man daher Alles zu vermeiden, was Blutandrang nach dem Auge und damit sowohl die sclerotico. choroiditis als den Druck im Auge befördern kann wohin namentlich das Arbeiten bei vornübergebogener Haltung gehört. Darauf kann ich nicht genug Nachdruck legen. Jede progressive Myopie ist drohend für die Zukunft. Wenn sie progressiv bleibt, so wird das Auge, während hinderliche Erscheinungen auftreten, schon bald weniger braichbar und nicht selten geht das Sehvermögen in dem 50. oder 60. Jahre, wenn nicht schon früher, unwiderruflich verloren, sei es durch Netzhautablösung, sei es durch Extravasat, oder durch Atrophie und Degeneration des gelben Fleckes.

Die Anzahl der von mir untersuchten Myopen beträgt mehr als 1500. Jedesmal wurde der Grad der Myopie genau bestimmt und notirt. Wenn nun der Myop sich nach Jahren oder Monaten wiederum anmeldete, dann wurde die Bestimmung wiederholt. So 
gelangte ich zu der Ucberzengung, dass die Myopie meistens einigermassen progressiv ist, dass dies zwischen dem 15-25. Jahre Norm ist, und dass die höchsten Grade oft die bedeutendste Zunahme der Myopie darboten. Abnatume der Myopie habe ich nie, weder in dem jugendlichen noch in dem mäunlichen Alter constatirt, es sei denu in denjenigen seltenen Fällen, in welchen die Myopie durch Krampf des Accommodationsapparates bedingt war -und nicht einfache Refractions. oder Accommodations-Anomalie vorhanden war. Auch in höherem $\Lambda$ lter ist es eine Seltenheit, dass der Grad der Myopie abnimmt. Das dioptrische System erfährt zweifelsohne in dem myopischen Auge dieselbe Veränderung, als in dem emmetropischen; wenn aber zu gleicher Zeit die Sehaxe an Länge zunimmt, wie dies sehr gewöhnlich in myopischen Augen zu geschehen pflegt, so wird diese Veränderung theilweise oder gänzlich compensirt und die Myopie kann auch sogar in höherem Alter progressiv bleiben. Dies Alles ist das Resultat von unmittelbarer Erlahrung, welche jedoch, in Bezug auf die einzelnen Individuen, nur einige wenige Jahre umfasst. Um üher den Verlauf der Myopie während des ganzen Lebens eine hinreichende Vorstellung: zu crhalten, musste daher auch die mitgetheilte Erfahrung von vielen Patienten zu Hülfe gezogen werden. Ihre Mittheilung wurde namentlich dann für zuverlässig gehalten, wenn sie durch die Uebergabe von solchen Brillen, welche früher für das Sehen in der Entfernung hinreichten, hestätigt wurde. Uebrigens hat es dabei natürlich nicht an Kritik gefehlt.

Wenn auf diese Weise der gewöhnliche Verlauf des Fernpunktes, das ist der Grad der Myopie. durch alle Lebenszeiten hindurch bekannt geworden, so war es nicht sehr schwierig, den Verlauf des Nahepunktes daneben zu stellen, wie in Fig. VI, VII u. VIII 
geschehen ist. Man braucht dazu nur die jeder Lebenszeit zugehörige Accommodationsbreite zu kennen. Ich bin in dieser Hinsicht zu dem Resultate gekommen, dass sie bei Myopen ungefähr dieselbe, wie bci normalen Augen ist. Bei den ersten grösstentheils von Dr. Mac Gillavry ausgeführten Bestimmungen schien. die Accommodationsbreite elwas grösser zu sein. Dies erklärt sich aber daraus, dass erstens unter den normaien :Augen Fälle von latenter Hypermetropie eingeschlichen sein mögen, deren Vorkommen uns damals noch entgangen war, und zweitens daraus, dass der lernpunkt der Myopen mit Hülfe von negativen Gläsern, bei parallelen Sehlinien, der Nahepunkt dagegen an unbewaffneten $\Lambda$ ugen bestimnt wurde, so dass der Unterschied in der Convergenz der Sehlinien grösser war, als an dem emmetropischen $\Lambda$ uge (Fig. IV). Die früher gefundene Accommodationsbreite bei dem Binocularsehen musste daher einigermassen reducirt werden, und man bleibt der Wahrheit gar nicht fern, wenn man die Accommodationsbreite hei mässigen Graden von Myopie mit der von normalen Augen gleich stellt. Bei sehr hohen Graden ist sie dagegen geringer. Dabei ist das ganze Auge und namentlich auch der vordere Theil mitsammt dem musc. Brïckianus ausgedehnt, was diess. Abnahme der Accommodation hinreichend crklärt.

Fig. VI, VII und VIII stellen drei Categorien von Myopie in ihrem am meisten vorkommenden Entwickelungsgang vor. Fig. VI ist eine stationäre, Fig VII eine zeitlich progressive, Fig. VIII eine bleibend progressive Myopie. Den Verlauf der Myopie deutet die Linie $r$ an, welche den Fernpunkt bei parallelen Sehlinien repräsentirt. Die jeder Lebenszeit eigene Accommodationsbreite*) ist durch die Entfernung

\#) Die Accommodationsbreite $A$, von welcher hier dic Rede ist, ist die für beide Augen gemeinschaftliche. Sie ist kleiner, als die 
der Linien $r \boldsymbol{r}^{\prime}$ und $p \boldsymbol{p}^{\prime}$ von einander gegeben. Ueber eine jede dieser Categorien will ich noch einige Bemerkungen anreihen.

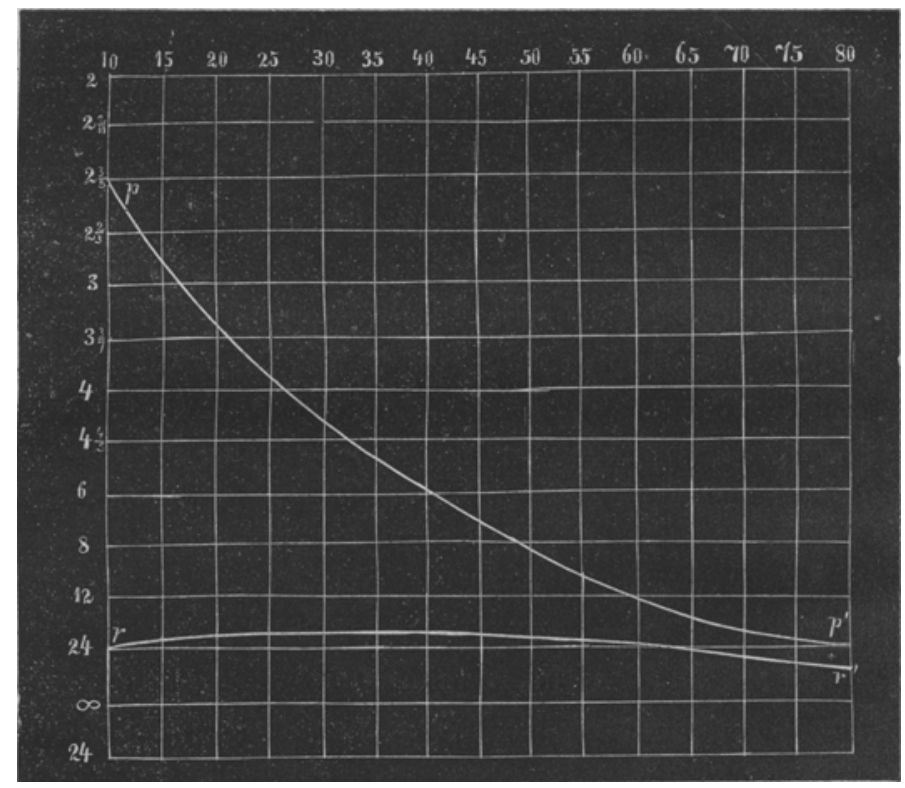

Figur VI.

Fig. VI wurde stationäre Myopie genannt. Und dennoch sieht man, wie die Myopie von $1 / 24$ bis auf. $1 / 16$

absolute Accommodationsbreite cines Auges $A^{\prime}$ (bei dem grösstmöglichen Wechsel der Convergenz) und steht zu derselben in keinem unveränderlichen Verhältnisse. $A$ ist nämlich um so kleiner in Beziehung zu $A^{\prime}$, je kleiner $A$ selbst ist, das heisst, je geringer der Convergenzunterschied ist, bei dem deutlich gesehen werden kann. Weiterhin aber wird $A$ auch bestimmt durch die grössere oder geringere Leichtigkeit, mit der convergirt wird und ist insofern von den äusseren Augenmuskeln abhängig. Endlich kann $A$ bei Schielenden, Einäugigen und bei grossen Untersehieden in dem Refractionszustande beider Augen gar nicht und bei Hypermetropen nur mit Anwendung einer Brille bestimmt werden. Trotzdem aber sind wir genöthigt, sobald wir nämlich einer gewissen Anzahl Beobachtungen bedürfen, uns in der Praxis auf 
steigt. Wie ich aber schon oben angah, ist derartiges Steigen in den Entwickelungsjahren ein nornaler Vorrang. Wenn mithin die Zunahme nicht bedeutender ist, so kann die Myopie im Gegensatze zur progressiven stationär heissen. Gewähnlich gehören die geringen Grade von Myopic hierher. Darum wurde auch fïr Fig. VI eive Myopie von nur 1/24 gewăhlt. Ein ursprünglich hoher Grad von Myopie kann aber auch stationär bleiben, und der geringste Grad von Myopie kamn auch bleibend progressip werden und hierdurch schliesslich einen sebr hohen Grad erreichen. Letzteres hat man namentlich dann zu beobachten Gelegeriheit, weun bei den Aeltern oder anderen Verwandten ein loher Grad rọ Myopie vorhanden ist, während übrigens die Lebensweise, namentlich das Sitzen in vornübergebeugter Haltung und das starke Convergiren der Sehlinien, die weitere Entwickelung der Myopie hefộrdern künnen. Bei dem günstigstén Verlaufe der Myopie (Fig. VI) bleibt sie im. Mannesalter ganz stationär; in späterem Alter kann sie sogar, wie die Figur angiebt, etwas abnehmen. Meistens geschieht dies aber nicht. Die allgemein verbreitete Meinung, dass die Myopie mit zunehmendum Alter abzunehmen pflegt, ist ein lirthum, weleher theils daraul beruht, dass man den Grad der Myopir durch den Nahepunkt bestimmen zu können glaubt, theils der richtig beobachteteten Thatsache

die für beide Augen Eemeinschaftiche Accommodationsbreite $A$ zu beschränken, weil die Bestimmung der absoluten $A^{\prime}$ in den meisten Füllen an unüberwindlichen Schwierigkeiten scheitert. Man kann die. Individuen nicht so weit bringen, dass sio bei dem Maximum der Convergenz. der Sehlinien eine brauchbare Wahrnehmnng mit einem Auge machen. Der Punkt $p^{\prime}$ in Fig. I, II und III, welcher den absolut näcbsten Punkt angiebt. ist dırch Bestimmung an einigen gut Geübten gefunden. - Die relative Accommodationsbreite $A^{\prime \prime}$, wovon in $\$ 5$ die Rede war; kann nur dann bestimmt werden, wenn beide Augen gleich und gut gerichtet sind. 
Eutstehung verdankt, dass allmälig deutlicher in grosser Entfernung geschen wird, was vielmehr durch die zunehmende Verengung der Pupille hedingt wird.

Die zeitlich progressive Myopie findet man in Fig. VII vorgestellt.

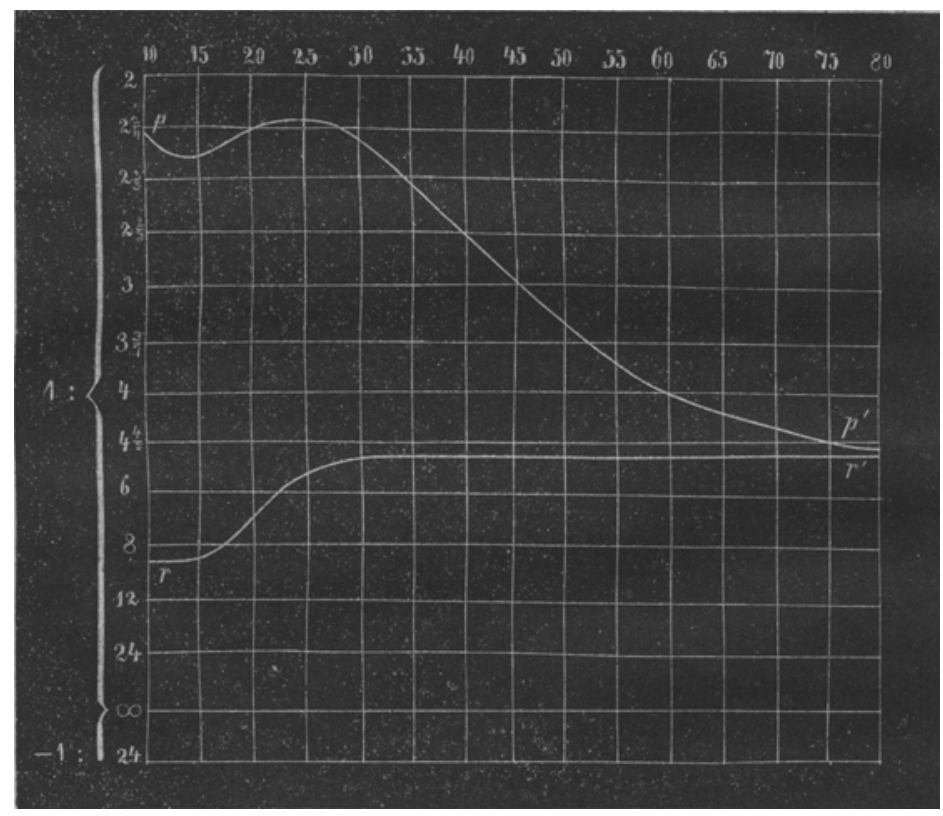

Figut VII.

Dio Progressivitäl latle meistans zwischen das 15. und 25. .lahr. lis gehürt zu den glücklichen Fällen. wenn die Myopie: wonigstens vor den 30. Jahre stationär wird. In Fig. VIl stright sie vou dem 13. bis zu dem 35. Jahne von $1 / x$ his auf $1 / 4$; las Steigen ist an geschwindesten von dem 18. bis z.1 dem 22. Jahre. Fandlich hleibt sie statiouär. Es ist als cine Ausuahm. zu betrachten; wenn sin stationär bleibt. nachdem sie eintnal diesen' Grad erreicht hat. Hohe Grade von Myopie scheinen niemals angeboren zu scin, wenn man Arrhiv fir Ophthalmologie. Vı. 2. 
anders nicht den angeborenen Buphthalmos hierher rechnen will. Ich wage es sogar nicht, zu eutscheiden, ob die Myopie, im Falle sie hereditär ist, stets auch schon in gewissem Grade angeboren ist. Ich glaube es kaum. leh habe hereditäre Myopie, welche im 12. oder 15. Jahre in sehr geringem Grade, z. B. 1/1s. vorhanden war, zu oft einen sehr hohen Grad von 1/4 oder 1/s erreichen sehen, an die Möglickeir zu läugnen, das sie in den ersten Lebensjabren fast ganz fehlte. Aut ler anderen Seite habe ich hörhst selten nach dem;15. und nie nach dem 20. Jahire Myopie entstehen sehen in Augen. deren Verhalten bis dahin ganz emmetropisch war. Es giebt zwar viele Augenkranke, welche meinen. dass ihre Myopie erst in dem Alter angefangen, aber nur deswegen, weil der primitiv vorhandene geringe Grad übersehen wurde. In diesem primitiven Grade, wie gering er auch sein mŏge, ist doch bereits der Keim gelegen. Die verschiedenartigen Klagen werden erst später gehört, wenn die Myopie progressiv wird. Sie progressirt an meisten, wenn sie im 17. Jahre sehon ziemlich bedeutend ist. z. $B_{+}=1 / 2$, wie es in Fig VII angenommen wurde. Der Verlauf in Fig. VII ist noch als ein relativ günstiger zu betrachten. Die Myopie bleibt selten ganz stationäl im Mannesalter; noch seltener geschieht es, dass sie in hohen Alter abnimnt. Oefter ist sie wenigstens einigermassen im Zunehmen begriffen und geht so über in die

bleibend progressive Myopie, welche Fig. VIII rurstellt.

In den meisten hierhergehörigen Fallen ist die Myopie im 15. Jahre schon sehr entwickelt. Darum wurde sie in dem in der Fig. VIII vorgestellten Falle $::=1 \%$ angenommen. Sie steigt am meisten bis in das 25., auch wohl 35. Jahr, langsamer in böherem. Alter, wir es: scheint unaufhörlich, aber doch öfter mit stoss. weisen Intervallen. Die Linie $r r^{\circ}$ stellt dies ïber- 


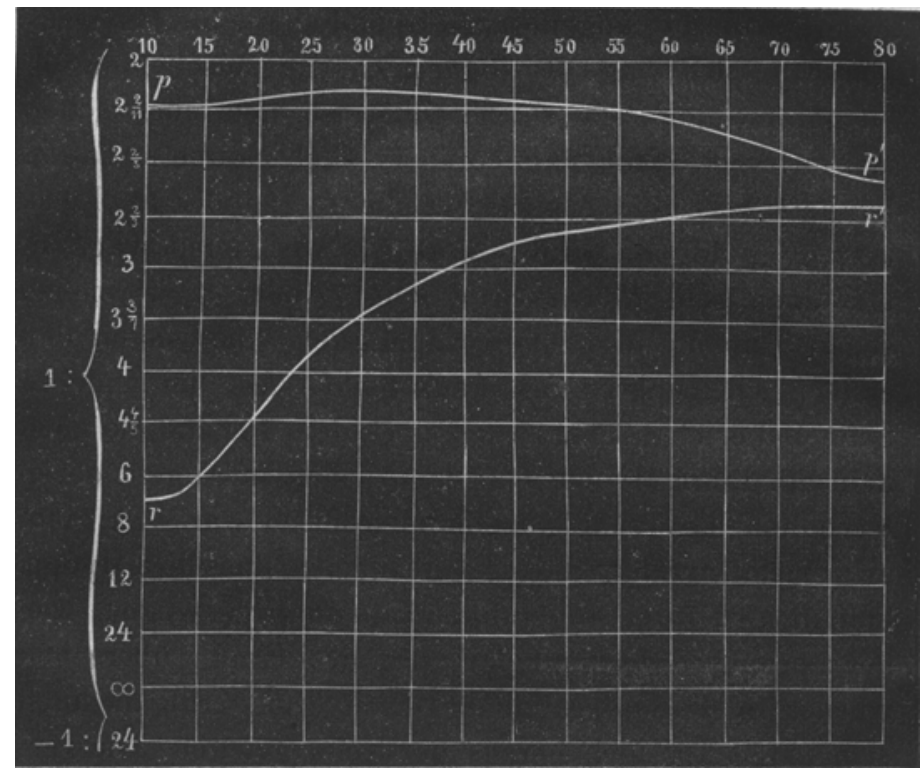

Figur VIII.

sichelich vor. Bie Myopie kann bis aul $1 / 2$ und sogar böher steigen. Dann ist aber auch das Schlimmste zu befürchten. Ks ist eino Seltenheit, dass man bei einer Myopie von $1: 2 \%$ oder sogar von $1: 3$ im 60 . Jahre ein ziemlich brauchbares Auge antrifft. Abuahme. von solchen Graden von Myopie in hohem Alter kanu niefut erwartet werden; der Winfluss von zunehmender Ausdehnung des Auges in der Richtung der Sehaxe wird nie übertroffen, ja nicht einmal compensirt durch die veränderte Refraction dor Ijinse. Solche Kranke werden meistens sehr traurig gestimm! indem sie empfinden, dass ihre Myopie fortwährend zunimmt. Die vo! Trïbungen im Glaskërper abhängigen mouches volantes heschäftigen sie fortwährend; hierzu kommen nicht selten Pbotopsien und scotomata, und viele dieser $U_{n}$ glürklichen verlässt der Gedank», dass totale Blindheit 
sie hedroht, fast keinen Augenblick. Dieser Gedanke wird mitunter zu piner idise lixe. auch schon dann. wenn noch kein hinleichender Grund datiij rurhanden ist. Bei diesem hohen Entwickelungsgrad hleiht der künstliche Blutegel von Heurteloup. Fussbfider und Drrivationen anderer Art erfolglos, kaltr Douclien werdron kaum ertragen. Dir unglïcklichen Patirnten findeh nork, am meisten Trost in dem Gebrauche von blauen, lïr das Sehen in die Ferne hinreichendev. die Myopre grüsstentheils neutralisirenden negativen Glïsern. Sit. unterscheiden dann elwas hessor und sind weniger ron den mouches volantes gerquält.

In ähnlicher Wrise wie die Myopie, wïnsehte irh auch die Hypermetropie in drci figuren vorzustellen.

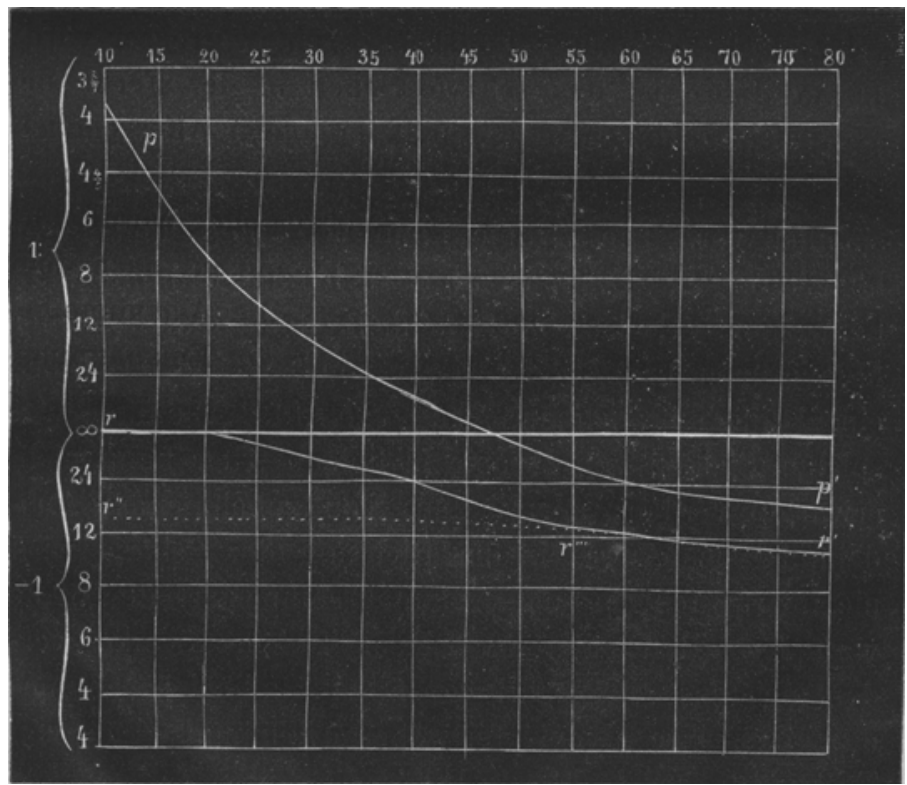

Figur IX.

Fig. IX stellt einen geringen Grad von Hypermetropie vor. Die Entfrrnung von $r r^{\prime}$ und $r^{\text {" }} \gamma^{\text {jo! ent- }}$ 
spricht dem latenten. erst bei Atropin-Paralyse bemerkbaren Theil der Hypermetropic, welche hei zunihmendem Alter im Verhältnisse zur Abnahme der Accommodätionsbreite allmälig abnimmt, endlich verschwindet, und dem entsprechend ersetzt wird durih manifeste Hyperruetropie, welcbe durch die Entfermung $\infty$ von der Linie $r$ angegebrn wird; die Entfernung $\infty$ von der Linie "r $r^{\prime \prime}$ stellt die totale Hypermetropi $=$ der Sunne der latenten und manifesten vor. Wenn der Grad der Hypermetropie gering wie in Fig. IX ist, so ist sie hei dem Kinde ganz. latent und wird erst in dem 20. Jahre manifest, indrm sir sich dureh scharfes und leichtes Sehen in der Ferne hoi dem Gebrauche von schwactien positiven (iläsern kund gịpt. Im 25. Jahre verursacht sie -Asthenopie, während die Hypermetropie bei abnehmender Accommodationsbreite relativ grösser wird; im 35. Jahre ișt das scharfe Sehen in der Ferne bei parallelen Sehlinion unmöglich; in hohem Alter endlich geht sic in absolute Hypermetropie über. Bei künstlicher Mydriasis, durch $\boldsymbol{r}^{\prime \prime} \boldsymbol{r}^{\prime \prime}$ vorgestellt, wurde unmittelhar $1 / 1$ "Hypermetropie wahrgenommen; nach dem 45. Jahre nimmt sie pin wenig zu. Die Accommodationsbreite vom nächsten Punkte an bis zur Entspannung bei Atropin-Paralyse berechuet, ist nicht viel geringer als in dem emmetropischen Augre.

Einen hïhrern Glarl von Hypermetropie finden wir in Fig. $\mathrm{X}$.

Bei Alropin-Paralyse rrejcht sie $1 / \%$; ohne diese Paralyse kann sie norh latent bleiben. Wenn aber pusitive Gläser auch nur während kurzer Zeit vor das Auge gehalten werden, so wird deutlich in die Ferne gesehen und die Ifypermetropie wird entderkt. Mit dem 20. Jahre ist jeder Zweifel hierïber schon greschwunden, und meistens trat die Asthenopie schon früher auf. Das Uebrige geht von selhst ans der Figur hervor. 


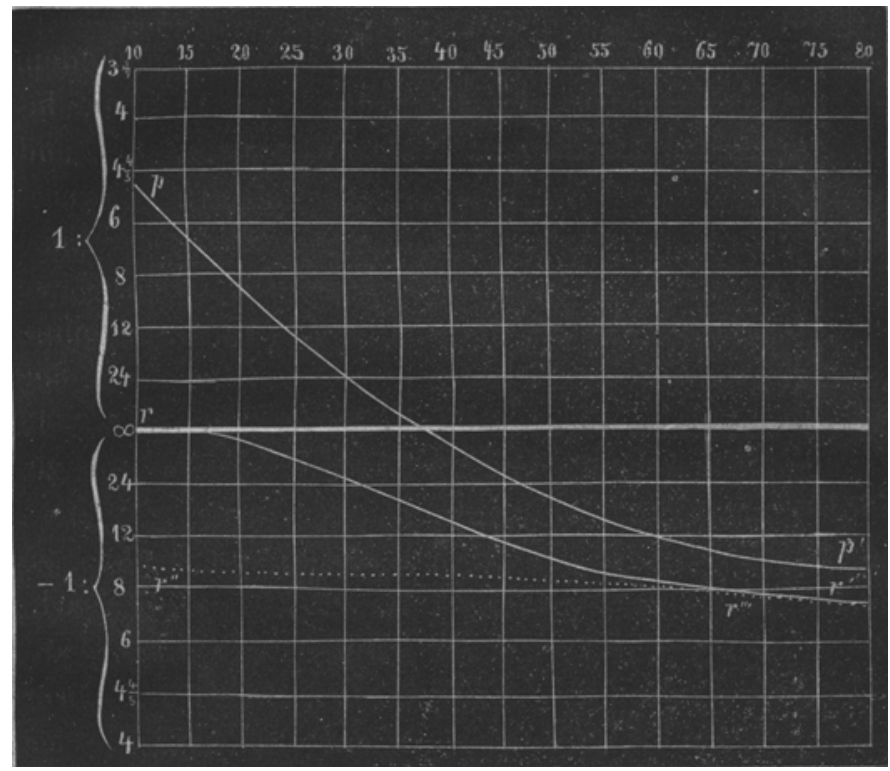

Figur $\mathbf{x}$.

Fig. Xil enthält eijusu hohen Girad won Hypermetropie. Sic haun, da sie von geringer Accommodationshreite begleitet ist. auch beim Kinde keinen Augrnbilick latent sein; mit positiven Gläsern wird besser in die Ferne geseben and kleine Gegenständ'e in grösserer Entfernung von dem Auge erkannt. Die geringe Accornmodationsbreite erfordert öfter schon in jugendlichem Alter den Gebrauch von zweicrlri Brillen, cine schwächere fïr die Entfernung und eine stärkere für die Nähe. Das Sehvermögen ist fast stets weniger scharf, als in dem emmetropischen Auge; in einigen Fällon liegt die UTrsache davon in der unegelmässigen form der Krümmungsfächen. Der fernere Verlauf des Nahe- und Fernpunktes in verschiedenem Alter weicht nicht sehr von dem im' emmetropischen Auge ab. Wenn $A$ in hohem Alter auf ein Miuimum redurirt ist, so hat das 


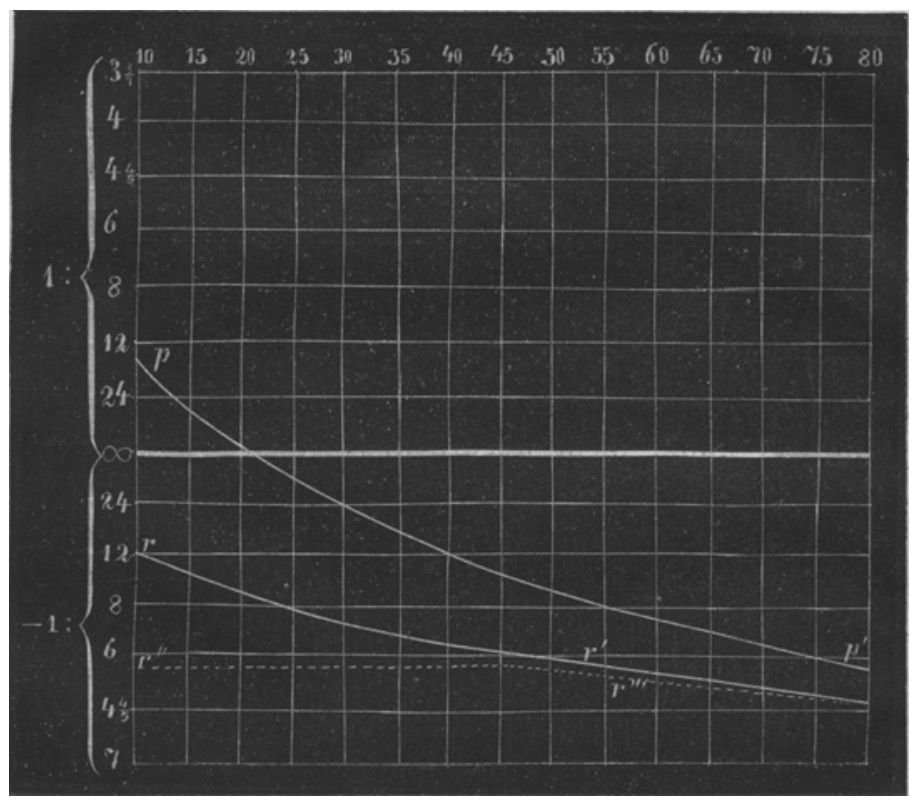

Figur XI.

Eintröpfelu vou Atropin kejuen Einfluss mehr auf die Lage des Fernpunktes, und die Linien $r r^{\prime}$ und $r^{\prime \prime}, r^{\prime \prime \prime}$ schneiden einander mithin.

$$
\text { Presbyopie. }
$$

In den vorhergehenden Paragraphen ist Alles besprochen, was zu dem Gebiete der Refractions-Anomalien gehört. Es kann nämlich - abgesehen von einer ausserordentlichen Asymmetrie des dioptrischen Systems - nur in zwei entgegengesetzten Richtungen Abweichung von dem emmetropischen Auge vorkommen, und die beiden dadurch bedingten Formen von Ametropie wurden als Myopie (Brachymetropie) und Hypermetropie behandelt. Es kann nun als natürliche Folge der Lebenszeit sowohl 
in dem ametropischen als in dem enmetropischen Auge die Accommodationsbreite vermindert sein, auch hierüber haben wir gehandelt: in dem vorigen Paragraphen kamen diejenigen Modificationen zur Sprache, welche von den Veränderungen der Brechungszustände des Auges bei zunehmendem Alter bedingt werden. Es sind terner keine Anomalien der Refraction denkbar. Was hat man demn unter Prosbyopie zu verstehen?

Presbyopie wird gleichgestellt mit Fernsichtigkeit. Man will damit nicht ausdrücken, dass das Auge in grosser Ferne schart sicht, denn auch das emmetropische Auge ist hierzu im Stamle. Man will nur damit sagen, dass es in der Nahe nicht schart sehen kanil. Ebenso gebraucht man den l'erminus kurzsichtig, nicht um damit anzugeben, dass Jemand kleino Gegenstände yanz nahe bei dem Auge unterscheiden kann - denn. diese Eigenschaft theilt das jugendliche emmetropisctie Auge mit dem kurzsichtigen -, sondern um anzudeuten, dass Jemand in grosser Entfermung nicht gut sieht.

Ich werde aber bei der Unrichtigkeit des 'Terminus Fernsichtigkeit nicht stille stehen, es kommt nur auf. den Begriff an und dieser muss näher bestimnt werden.

In dieser Hinsicht muss zuerst hervorgehoben werden, dass nur diejenige Fernsichtigkeit als Proshyopie zu betrachten ist, welche von Abnahmo der Accommodationsibreite in Folge vorgeschrittener Lebenszeit bedingt wird. Dies deutet schon die Etymologie des Terminus

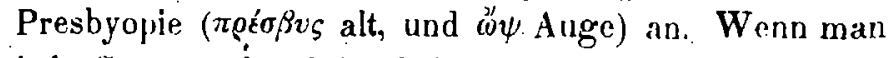
jede Störung des Scharfsehens in aler Nähe Prastiyopie nennen wollte, so würde auch die Lähụung des Accommodationsvermögens dahin gerechnet werden müssen. Selhst die Hypermetropie würde nicht ausgeschlossen sein, insofern dabei leichter in der. Ferne als in der Nähe gesehen wird; in $\$ 2$ aber haben wir schon angegehen, welche grosse Verwirrung von Begriffen dalaus 
entstehen wïrde. Presbyopic kamn daher nur der Zustand heissen, bei dem in Folge zumbhmenden alurs die Accummordationsbreite geringer und das Sehen in ther Nathe schwieriger geworden ist.

Avis dieser Definition geht aber hervor, dass die Presbyopie wirklich schon in dem enthalten ist, was in der vorigen Paragraphen abgehandelt worden ist. Denu Presbyopie ist die normale Eigenschati des nurmalen (emmetropischen) Auges in hïherem Alter. Sis ist daher keine eigentliche Anomalie, ehensowenig wie graus Haare oder gerunzelte Haut.

Wann fängt aber die Presbyopie an?' Wem man die' I.jirie $p$. $\boldsymbol{p}^{\prime}$, Fig. XII, welche das emmetropische Ange in verschiedenem Alter vorstellt, näher betrachtet, so sieht man, dass dec Nahepunkt von der Jugend an bis zum hohen Alter sich zienlich regelmâssig melir und: mehr ron dem Auge entfernt und dass daher das Sehen in der Näho stets schwerer wird. Ein Rưhezustand kommt in der Linie nicht vor. Daher nun die Schwierigkeit, einen Grenzpunkt der Presbyopie zu finden. Die Linie befähigt uns, für jedes Lebensjahr die Entfernung des Nahepunktes genau zu bestimrnen; wenin wir nun auch den durch die Linie $r$ vorgestellen Fernpunkt des deutlichen Sehens kennen, so wissen wir Alles, was wir nöthig haben, um die Refraction und Accommodation des Auges zu beurtheilen. Zur Bestimmung aber des Anfanges der Presbyopie giebt diese lirie auch nicht den geringsten Aufschluss.

Hieraus folgt, dass die Annatıme einer Gienze ziemlich willkürlich ist. In dem Auge selbst ist keiu Grund z.ur scharfen Trennung von presbyopisch und uicht preshyopisch vorhanden. Wenn die Grenze mithin küntlich ist, so muss sie conventionell sciu.

Dies führt uns aber zu dor Frage, ob es wohl nöthig sei, eine Preshyopir anzunchmen und ob es nicht 


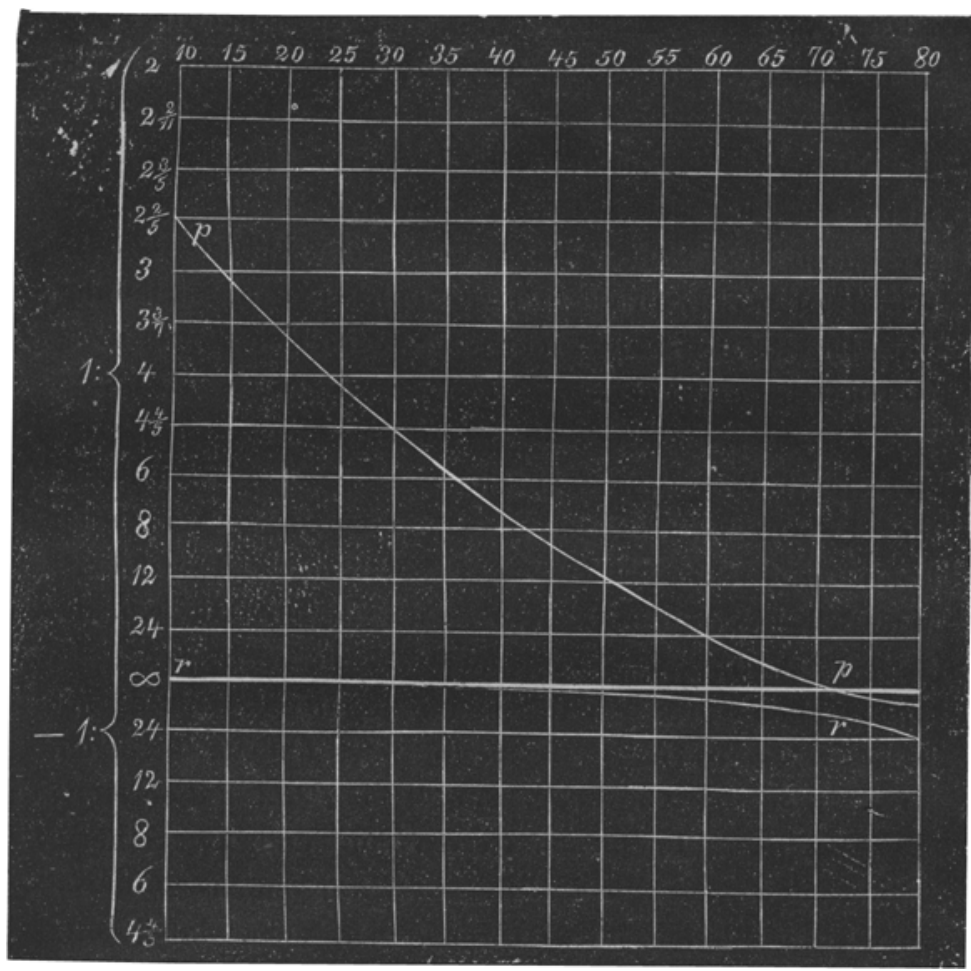

Figur XIJ.

besser wäre, es bei der Bestimmung der Accommodationsbreite und des Grades der Myopie oder IIypermetropie, wo sie vorhanden sind, bewenden zu lassen. Dies würde zweifelsohne wissenschaftlicher sein. Man würde aber trotzdem nicht auf grosse Sympathie rechnen können, wenn man einen so allgemein bekannten und gebrauchten Terminus abschaffen wollte. Ich glaube auch, dass der Praxis hiermit wenig geholfer whre. Sic bedarf eines Wortes, um den Zustand anzudeuten; bei dem das Auge in vorgeschrittenem Alter für Arbeiten in der Nähe einer positiven Brille bedarf, und dieses Wort ist Presbyopie, 
Damit ist aber der Anfang der Prosbyopie noch nicht näher bestimmt. Es ist aber klar, dass dies nicht unterlassen werden darf.

Unsere gesellschaflichen Zuständo bringea es mit sich, dass wir uns mit Lesen, Schreiben oder anderer Arbeit in der Nähe beschäftigen. Die mittlere Grösse der dazu benutzten Formen steht of'enbar in Beziehung zur Schärfe des Sehvermögens und zu der Entfernung drs deutlichen Sehens für das normale Auge. Dasselbe gilt für die Kunstproducte, sowie für eine Anzahl Handarbeiten. Was das menschliche Auge in der Blüthe des Juebens vermag, ist im Allgemeinen als Maasstab dalür angenommen. Vor der allgemeinen Anwendung der Brillen war dieser Maassstab zweifelsolne ein anderer. Wenn diese Instrumente nicht mehr so leicht zu ertualten wären, so würde im Allgemeinen wiederum eine grössere Druckschrift die jetzt übliche ersetzen. Der allgemeine Gebrauch von Brillen hat daher einen Winfluss ausgeübt auf die Grenze des deutlichen Sehens, mit welcher man die Preshyopie anfangen lassen muss. Das Unbestimmte dieser Grenze tritt eben dabei auts deutlichste hervor. Wir haben nun zu untersuchen, wie lange das Auge den Forderungen des angenommenen Maassstabes Geuïge leistet. Schon im 30. Jahre liest das normale Auge oft ungern die kleine Druckschrift, welche der Kurzsichtige vorzüglich auswählt und welche die Jugend nicht vermeidet. Im 40. Jahre aber bietet die üblicbe Druckschrift durchaus noch keinie Schwierigkeit für das emmetropische Auge. Mit dem 45. Jahre werden die mit kleinen Buchstaben gedruckten Aumerkungen nicht selten unbeachtet gelassen und das Buch vielleicht am Abende etwas früher zur Seite gelegt. Bald darauf nun făngt man an zu bemerken, dass ein Gegerstand etwas mehr von dem $\Lambda$ uge entfernt wird. wenn er sehr scharf gesehen werden soll; alch:wird 
das helle Licht gesucht, mehr noch un die Zerstreuungskreise bei unvollkommener Accommodation durch Verengung der Pupille kleiner zu machen, als um hell beleuchtete Bilder zu erhalten. Indessen wer: den die am Abende gewöhnlich vorgenommenen Arbeiten noch lange hintereinander ohne bemerkbare An: strengung fortgesetzt. Wo es aber gilt, mitunter vorkommende feinere Objecte recht schart zu sohen, da unuss man, wie ungerne auch, dio Klage laut werden lassen, dass die Augen nicht mehr sind, was sie zuvor waren. Der Nahepunkt liegt nun meistens il .eciner Entternung von 8" von dem Auge. Den Antang der Pres: hyopie habe ich schon früher an dieser Grenze angenommen, und glaube auch jetzt noch daran festhalten zu müssen. Man lejte daraus aber nicht her. dass der Gebrauch einer Brille unmittelbar anzurathen sei, wenn der Nahepunkt mehr als $8^{\prime \prime}$ von dem Auge entfernt ist. Es. kommt doch nicht selten vor, dass normale Augen sich am Tage und Abends noch Stunden lang mit Lesen und Schreiben beschäftigen, ohne dabei eine besondere Ermüdung zu empfinden, obgleich der Nahepunkt schon bis auf $12^{\prime \prime}$ oder $14^{\prime \prime}$ von dem Auge entfernt ist. Dazu aber muss das Sehvermögen ausserordentlich scharf sein. In gewöhnlichen Fällen ist eine schwach positive Brille, wenigstens Abends, schon sehr wohlthätig, wenn erst der Nahepunkt in einer Entfernung von 10" oder 11" gelegen ist, nicht selten auch schon früher. Wenn aber das Auge die wohlthätige Wirkung empfindet. da ist das Wünschenswerthe des Gebrauches über jeden Zweifel erhaben. Das subjective Gefühl ist hier vollkommen entscheidend. Dem Vorurtheil, dass man sich bei Entstehung von Pres: byopie so lange als möglich des Gebrauches einer Brille zu enthalten habe, kann man nicht kräftig genug entgegentreten. 
Indem die Lage des Nahepunktes, bei dem dje Unterstiizung durch Brillengläser wïnschonswerth wird, individuellen Unterschieden unterliegt, so wurde dessen geringste Entfernung gewählt, um den Anfang der Presbyopie :zu hestimmen. Lir wurde laher auf $8^{\prime \prime}$ gestellt. loh wünschte indessen nicht gertu, dass os so verstanden würde; als oh grade mit dieser Enternung absolut die heste Grenze angegeben' wäre; im (jegentbeile, ich wiederhole es, dass main hierbei ziemlich willkürlich verfahren kann, und ich mache dahrer uur eiuen Vorschlag. Nöthig ist es aber. dass man sich über eine gewisse Entfernung verstehe, damit auch der Grad der Presbyopie näher bestimnıt werden könne. Dies geschinht nun auf selur einfache Weise. Wenn nämlich der Nahepunkt in der Entfernung von n Pariser Zoll von dem Auge gelegen ist; so ist, wenn man die vorgesteltte Grenze annimmt, die Presbyopie $=1 / \mathrm{s}-1 / \mathrm{n}$ Wenn also der Nahepuntit auf 16 Zoll Entfernung

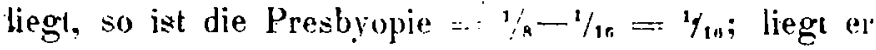
auf $24^{\prime \prime}$, so ist sie $=1 / n-1 / 24=1 / 12$. Dabei denn werden Gläser von $1 / n-1 / n$, und in den angeführten Beispielen Gläser von $1 / 1$, und $1 / 1$ nö̈hig sein, um den Nahepunkt auf" 8 " zu bringen und so die Preshyopie zu neutralisiren. In der Regel kann man aber schwächere Gläser geben, weil einestheils der Nahepunkt bei der grösseren Convergenz der Sehlinien durch die erwähnten Gläser näher als $8^{\prime \prime}$ wird. und weil anderentheils das scharf sehende Augre, wie wir oben sahen, fast allen Forderungen genügt, wenn der Nahepunkt. auf 10 bis $12^{\prime \prime}$ geloracht wird. Ich würde sehr ausführlich sein müssen, wenn ich fïr alle vorkommenden Fälle hier die nöthige Anweisung geben wollte. Nur dir Praxis kann uns die richtige Anwendung dessen lehreu. was hier im Principe angegeben wird. Man liann aher als leitende Regel annehmen. dass der Nahepunkt. 
wenn die Gesichtsschürfe vermindert ist, wie dies fast ohne Ausnahme in den 70or Jahren geschieht, dom Auge durch die Brille näher, mitunter auf 7 " oder 6 “. gebracht werden muss, und weiter, dass der Nahepunkt lem Auge um so näher gebracht werden muss, je grösser die Accommodationsbreite ist. In letzterem Falle (an meisten bei Hypermetropie von jungen Leuten) liegt nämlich die Mitte der Accommodationsbreite, deron mun sicli vorziliglichtiedient, ziemlich weit von d'om Nahepunkte nntfurnt. Endlich darf ich hinzufügen, dass diebchwäh harn Gläser nit denen in der Entfernung ungetähr eines Fusses Vr. 1 von Jäger s Druckproben gelesen wird. wenu keine Hypermetropie vorhanden ist, in del Regal ganz. hinreichend befunden werden.

Bis dahin wurde nur über dic Presbyopic des emmetropischen Auges gehandelt. Alier auch das hypermetropische und das myopische Ange kümren preshyopiseh werden. 1)as Fistere wird rs, sobald der Nahepunkt bei rem Gebrauch von Brillen; welche die Hypermetropie neutralisiren; ferner von d'm Auge liegt, als $8^{\prime \prime}$. Dies findet nun fast früher statt, als in dem emmetropischen Auge. Die Ursache dafïr liegt nahe: sie ergiebt sich aus der geringeren Accommodationsbreite. welehe dem lyjpermetropischen Auge meistens zukommt. Sie ist nirht selten von einer geringeren Schärfe des Sehvermögens beglcitet. wodurch die Preshyopie um so eher empfunden wird. Sohald daher der Nabepunkt bei Hypermetropen mit reducirender Brille mehr als $8^{\prime \prime}$ von Auge eritfornt ist, werden stärkere Gläser für Arbeiten in' der Nähn' im Allgromeinen erwïuscht sein. Sie haben alsdarin zwei Brillen nöthig: eine, welche die:Hypermetropie neutralisirt für das Sehen in der Entfernung; eine andere, welche um so viel stärker als der Grad der Presbyopie, für das Lesen, Schreiben .u. s. w. Auch Myopen können, wie ich angał), preshyopisch, 
werden. Wenn wir uns an die gegebene Definition dor Presbyopie halten, so fängt sie bei dieser ebenfulls an, sobald die Entfernung des Nahepunktes mehr als $8^{\prime \prime}$ beträgt. Daraus geht hervor, dass Presbyopie nur bei geringen Graden von Myopie vorkommen kann, dass sie bei einer Myopie $=1 /$, schon ausgeschlossen wird. -isogar bei totalem Verluste des Accommodationsvermögens. Wir mïssen hinzufügen, dass sie bei den geringen Graden von Myopie viel später eintritt, als in dem-emmetropischen Auge. Hierin findet der Myope eine Compensation für dasjenige, was ihm beim Sehen in der Ferne abgeht. Der Vortheil ist nicht unbedeutend. Es ist doch ein grosses Vorrecht, Alles was unter die Augen kommt, im 60. oder 70: Lebensjahrs: ohne Brille unmittelhar scharf zu sehen. Dieses Vorrecht geniesst ein Myop von $1 / 10-1 / 14$, bei welchem Grade das Auge noch durch keine besondere Gofahren bedroht wird. Bei Myopie in geringerem Grade kommt Einem schon ein bedeutender Theil davon zu Gute. Für emmetropische Augen ist dies ein heneidenswerther Zustand. Nie fand ich ein normales Auge, dem ein solches Vorrecht zu Theil ward. Vicle aber sind von diesem glitcklichen Wahn befangen. Beinahe täglich kommt es vor, dass im 55. Jahre die Entfernung des Nahepunktes auf 8 bis $10^{\prime \prime}$ liegt und noch an keine Brille gedacht wird. Solche meinen, sie wären glïckliche Ausnahmen. Die Frage, ob sie kurzsichtig sind, wird lächelnd mit Selbstwohlgefallen verneinend beantwortet. In einer Entfernung von 6 Meter härigen aber. Buchstaben: Nr. 19.20 von Jäger's Druckproben, dazu noch die grösseren bis und mit Nr. 24; Nr. 19 lesen sie nicht; Nr. 20 nicht oder wenigstens mit grosser Schwierigkeit; erst 21 und 22 liegen in ihrem Bereiche. Gläser von $1 / 50-1 / 30$ bewirken erst, dass sie die Buchstaben von Nr. 19 in scharfen Contouren gut erkenmen. Nur ungerne erklären 
sie sich besiegt. Sie sind denn duch uinigermassen myopisch! Wahr ist es, dass sie dem Begriffe der Myopie eine ganz andere Bedeutung beigelegt haben. Für den Augenarzt aber ist es wichtig, diesen geringen Grad von Myopie constatirt zu habeu. Er lernt daraus das Unveränderliche, Gesetzmässige der jedem Alter eigenen Areonmorlationshreitr: kennon. Und diese Kenntniss ist ihm sehr nitzlich. So wird gewöhrulich dir Existenz der Myopie hei den Eltern, im Falle wir über die Herediät der Myopie nachlorschen, negirt. aher in einem Athem hinzugreügt, dass sic bis in das 50. Jahr. ja länger noch, ohne Brille lesen und schreiben kounten - und man weiss, woran man sich zu halten hat. Wenn sich dagegen. Jemand zu uns verfïgt, der im 35 . oder 40 . Jahre einer positiven Brille bedarf, um seine Arbeit in ler Nähe fortzusetzen, so kaun man fast mit. völlige! Sicherbeit daraus schliessen, dass hier rin geringer Grad von Hypermetropie mit unterläuft. Wäre der Grad ein 'mehr bedeutender, so hätle sich diese Schwierigkeit schon früher und zwar unter dem Charakter der Asthenopie noch deutlicher offenbart. - Je mehr ich untersuche. um so mehr befestigt sich bei mir die Ueberzeugung, dass die Accommodationsbreite in einen gegebenen Lchensalter cine l'ast gesetzmässige Grüsse ist.

Man hört öfters den uralten Gebrauch, die convexen Gläser nach der [jebenszeit, bei der sie nöthig werden, zu orduen, bespöttelu. Es ist denn auch wirklich Jächerlich. Die Augen siud doch zu verschieden, als dass nur das Alter dic Wahl einer Brille entscheiden könnte. Für cmmetropische Augen ist indessen wirklich das Alter ein fast unfehlbarer Wegweiser. Sind aber die Augen nicht emmetropisch, so ist nichts leichter, als den Grad der Myopie und sogar den 
der Hypermetropie zu bestimmen - denn von latenter Hypermetropie kann kaum noch die Rede sein, wenn Presbyopie im Entstehen begriffen ist. Wenn man nun die tür ein gewisses Alter bei normalen Augen nüthigen Gläser nach dem Grade der gefundenen Myopie oder Hypermetropie modificirt, so werden sie, meiner Ueberzeugung nach, beinahe ausnahmslos passend befunden werden. Kennt man mithin das Alter und den Grad der Myopie oder Hypermetropie (die Behauptung gilt wenigstens für mässige Grade) so ist die Stärke des erforderten Glases nahe genug bestimmt.

Wie oben schon angegeben ist, kann ein Auge mit Myopie in höherem Grade nie presbyopisch werden. Es verliert an Accommodationshreite mit dem Zunehmen der Jahre; der Nahepunkt entfernt sich mehr und mehr ron dem $A$ uge und dies kann auch mit dem Fernpunkte stattfinden; die physikalischen Veränderungen, welche dabei im myopischen Auge stattinden, sind denen in anderen Augen durchaus äbnlich. Presbyopie darf hier der Zustand aber doch nicht beissen, weil der Nahepunkt sich nicht mehr als 8 " von dem Auge entfernt. Hieraus geht wiederum das Willkürliche, Conventionelle des Begriffes Presbyopie hervor. Ich will es gar nicht verbehlen, dass ich anfangs geneigt war, dem Terminus Presbyopie eine mehr ausgedehnto Bedeutung beizulegen. Ich wollte damit die jedem Auge zukommende senile Veränderung ausdrücken. „Diese Veränderung,” so war mein Gedankengang, "durch bestimmte anatomische Eigenschaften charakterisirt, kommt in jedem Auge ohne Unterschied zu Stande. Sie veranlasst Siörungen im Sehen und zwar dieselben bei jeder Form des Auges: das Auge nämlich kann weder unbewaffnet, noch mit gewissen Gläsern versehen, willkürlich in der Ferne oder in der Nähe scharf unterscheiden. Diese 
dem Alter eigenthümliche Störung verdient den Namen Presbyopie."

Vom wissenschaftlichen Standpunkte betrachtet, ist diese Anschauung ganz richtig. So lange Presbyopie im Gegensatze zu Myopie gestellt wurde, konnte diesem Terminus keine ausgedehntere Bedeutung beigelegt werden: denn Myopie musste Presbyopie ausschliessen, und die Presbyopie konnte nicht bei alten Myopen vorkommen, so bedeutend auch der Grad der senilen Veränderung war. Jetzt aber, da dieser Gegensatz nicht mehr besteht, sieht man leicht ein, dass das myopische Auge auch presbyopisch werden kann, und es drängt sich uns als von selbst der Gedanke auf, jede senile Veränderung mit verminderter Accommodationsbreite in jedem Auge ohne Unterschied mit dem Terminus Presbyopie zu bezeichnen. Ueherdies spricht auch die Etymologie dafür, dem Worte Presbyopie eine ausgedehntere Bedeutung zu geben. Denn Fernsichtigkeit kommt in der Regel bei alten Leuten vor und darum hat man sie Presbyopie genannt, mit mehr Recht noch könnte dieser Terminus mit dem $\mathrm{Zu}$ stande, der von hohem Alter untrennbar ist, verbunden werden.

Auf der anderen Seite: verba valent usu. Dies hat schliesslich schwerer bei mir gewogen, als die Forderungen der Logica und der Etymologic. Dabei dachte ich daran, dass die Praxis eines Wortes bedarf, um anzudeuten, dass übliche Beschäftigungen in der Nähe schwerlich ohne optische Unterstützung ausgeführt werden können und dafür wollte ich nicht gerne wiederum einen neuen Terminus vorschlagen. Darum babe ich das Wort Presbyopie in seiner gangbaren Bedeutung beibehalten. Nur der Begriff wurde gereinigt. Es wurde Alles, was zur Hypermetropie und Paralyse getürt, 
daron getrennt; die senile Veränderung mit Abnahme der Accommodation wurde in jedem Falle als Kennzeichen angenommen, und von diesem Gesichtspunkte aus war es nicht schwer, die Grenzen der Anwendung dieses Terminus auf myopische und hypermetropische Augen zu bestimmen. 